\title{
Tracking particles in Poiseuille flow for several pipe diameters in three dimensions
}

\author{
S. Sridharan, C. Poelma \\ Department of Mechanical, Maritime \& Materials Engineering, Delft University of Technology, Delft, The \\ Netherlands
}

\begin{abstract}
A setup is devised to track suspended particles in a pipe in three-dimensional space using the ShadowgraphyPTV technique. This system consists of a single camera and a mirror, and is used to track particles for over 20 pipe diameters at three downstream locations. Pipe to particle diameter ratios (D/d) of 18, 9, and 6 are investigated. The bulk Reynolds number is varied between $R e_{b}=300-1250$. As expected, particles are observed to migrate radially to a location corresponding to the Segré-Silberberg annulus. In addition, we observe particles also moving in the azimuthal direction (clockwise or counter-clockwise), with some particles moving as much as $180^{\circ}$ during their passage through the field of view. This helical motion persists throughout the pipe (600D long) and the azimuthal velocity increases with the Reynolds number $\left(\operatorname{Re}_{b}\right)$. The effect of particle size and the Reynolds number on this previously undocumented, three-dimensional motion is studied.
\end{abstract}

\section{Introduction}

Particles suspended in Poiseuille flow undergo inertial migration to a particular radial equilibrium position, commonly referred to as the Segré-Silberberg annulus. The dominant contribution to this effect is the lift force due to shear around a particle, following from the parabolic velocity profile (Asmolov, 1999). Matas et al. (2004) showed that increase in size and/or Reynolds number results in accumulation of particles at an additional radial location, more towards the pipe centre. The long-term stability of this 'inner annulus' is not clear, as the theory (which neglects finite-size effects) predicts that the particles should migrate even closer to the wall as $R e_{b}$ increases. However, a larger fraction of particles are observed to reach the inner annulus at higher Reynolds numbers 1000D away from the entrance (Morita et al. (2017)). Recent experimental work from Nakayama et al. (2019) show that there is a critical Reynolds number above which the inner annulus is more stable. It should be noted that previous experiments are based on stochastic analysis of particle radial locations, i.e. only based on locations in a cross-sectional plane at various streamwise locations. Modelling this effect is challenging even at single particle level when inertia becomes significant, especially when the particle size is very high $(D / d<15)$ (Hood et al. (2015).

In order to investigate these phenomena in more detail, we track particles over several pipe diameters in three dimensions with a measurement technique based on shadowgraphy and particle tracking velocimetry. The flow is imaged along two perpendicular directions, and particles are detected by their shadows. The 3D position is reconstructed from the two projections by matching the downstream coordinate of each particle. The magnification is adjusted based on the particle size to ensure consistent detection, allowing larger field of views $(L / D \approx 20)$. This is preferable as we intend to locate large particles slowly migrating over a long distance: typically cross-stream velocities are less than $5 \%$ of its streamwise velocity. Based on our observations, particles are observed to accumulate at two radial locations $(\approx 0.8 \mathrm{R}$ and $0.6 \mathrm{R}$ from pipe axis $)$ at higher Reynolds numbers $\left(\operatorname{Re}_{b}>700\right)$, with larger particles $(D / d=6)$ preferably staying at the inner location. This persists even 500 pipe diameters away from the entrance. Further, particles with $D / d=6$ and 9 exhibit peculiar spiralling behavior, i.e. they also have an azimuthal velocity if their radial location is more than $0.4 \mathrm{R}$ from the center. 


\section{Experimental Setup}

A flow loop consisting of a $6.5 \mathrm{~m}$ long pipe with $0.01 \mathrm{~m}$ diameter (D) is constructed, see Fig. 1 for a schematic representation. The flow is driven by a constant head maintained by an overflow tank. The suspension enters the pipe through an inlet chamber containing a flow conditioner, followed by a smooth contraction. The outflow of the suspension from the pipe is collected in a reservoir and pumped back to the overflow tank using a peristaltic pump, forming a closed loop. The bulk Reynolds number $\left(R e_{b}=U_{b} D / v\right)$ is maintained between 300-1250. A neutrally buoyant solution is prepared by adding Sodium sulfate to the water to match the density of the Polystyrene particles $\left(\rho_{p}=1035 \mathrm{~kg} / \mathrm{m}^{3}\right)$. Particles with a mean diameter of $0.515 \mathrm{~mm}, 1.12 \mathrm{~mm}$, and $1.67 \mathrm{~mm}$ were used, corresponding to D/d ratios of 20,9 and 6 respectively. The particle Reynolds number $\left(\operatorname{Re}_{p} \equiv \operatorname{Re}_{b}(d / D)^{2}\right)$ varies from 1.2-35. The particle concentration is kept below $0.05 \%$, typical spacing between the particles for $\mathrm{D} / \mathrm{d}=20,9,6$ are $0.6 \mathrm{D}, 1.5 \mathrm{D}$, and $10 \mathrm{D}$ respectively. At these concentrations, the suspension kinematic viscosity is very close to that of pure water $\left(\mathrm{v}=1.1 \times 10^{-6} \mathrm{~m}^{2} / \mathrm{s}\right.$, at $\left.20^{\circ} \mathrm{C}\right)$.

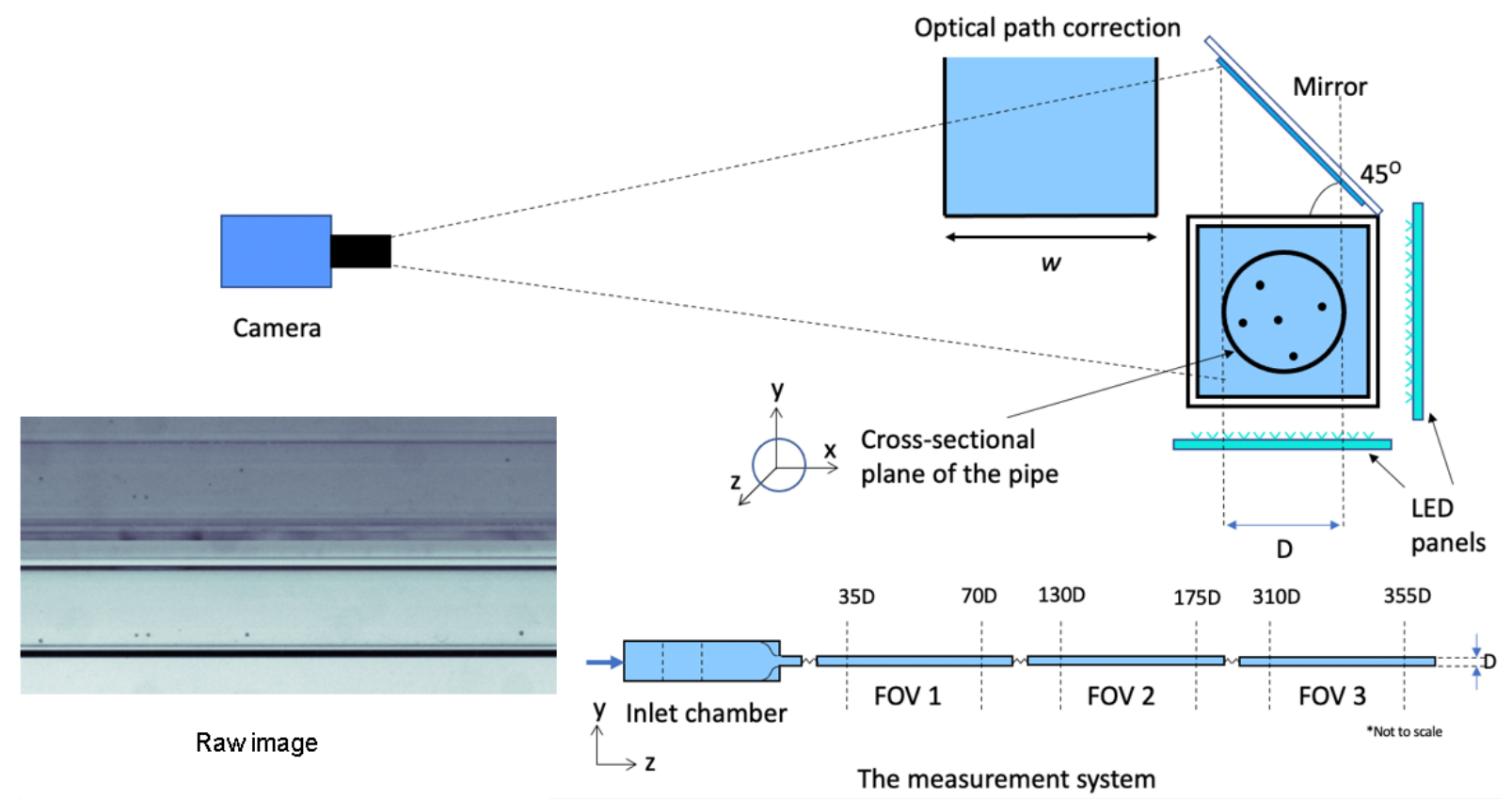

Figure 1: Schematic diagram of the optical system used to reconstruct the three-dimensional position of a particle in the pipe in the cross-sectional $(x-y)$ plane. The particles are tracked in three field of views: FOV 1, FOV 2, and FOV 3 corresponding to 35-70D, 300-350D, and 510-550D from the pipe entrance, respectively. A typical raw image is shown with top view and front view of the pipe.

The imaging system consists of a camera and a planar mirror positioned at $45^{\circ}$ to the object plane $(y-z$ plane, Fig-1). A water-filled container with calculated width is used to compensate for the optical path difference between the reflected and direct paths. The system is arranged such that the planes along the perpendicular views intersect at the axis of the pipe. This allows reconstruction of any point inside the pipe into a 3D space. An Imager sCMOS camera (LaVision $\mathrm{GmbH}$ ) with $50 \mathrm{~mm}$ lens is used, along with two LED panels placed perpendicular to the $(x-y)$ plane. A mirror is placed at $45^{\circ}$ with the horizontal $(x$ axis) above a rectangular PMMA section enclosing the pipe. A typical image contains two perpendicular views of the pipe in the $(y-z)$ and the $(x-z)$ planes with the $z$ axis as the epipolar line. The field of view is varied depending on the particle (image) size, covering $10 \mathrm{D}, 20 \mathrm{D}$, and $45 \mathrm{D}$ of pipe length for $\mathrm{D} / \mathrm{d}=18,9$ and 6 respectively. The images are captured at three downstream locations: 35D-70D, 300D-320D, and 500-510D from the entrance. The images have a spatial resolution of $0.22 \mathrm{~mm}$ and a temporal resolution of $0.02 \mathrm{~s}$. Particles are tracked separately in individual views and the tracks are reconstructed in 3D space by matching their streamwise locations. Occlusions/ghost particles are rare occasions at the concentration studied. Also, the relatively linear behaviour facilitates tracking in each view and allows matching based on first order estimates (i.e. constant displacement for each particle across frames). There is a small mismatch in streamwise locations between the two views because of the difference in their optical path $(\Delta s)$ away from the image centre as the correction is only valid at the image centre. This introduces a linear deviation in 
streamwise locations between the views with a difference of $\Delta s \tan \theta$, where $\theta$ is the viewing angle (along $z$, see Fig. 1). Further, there is an additional difference in depth of particle between the views due to its location in 3D space. From our results, their mismatch varies linearly with $z$, with a maximum of $40 \mathrm{px}$ towards the end of the image, corresponding to $2.6 \mathrm{~mm}$. The mismatch error is corrected by a self-calibration function based on the cross stream positions of multiple particles tracked in both views at very low concentration (Fig. 2). This function is used to correct the streamwise position of particle images in the top view and are further matched with the images in the front view to reconstruct their 3D trajectories (Fig. 8).
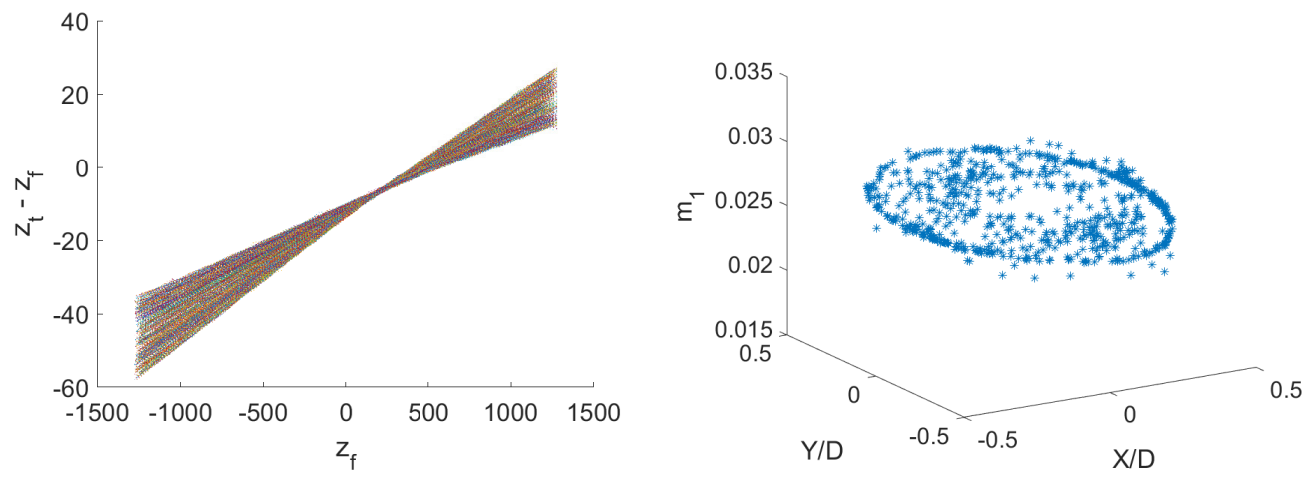

Figure 2: Mismatch in streamwise (z) coordinates of particles between both views (left), where $t$ and $f$ subscripts represent top view and front view respectively. The slope of the linear mismatch (right) is a function of the cross stream positions.

\section{Results}

The particle cross stream positions are used to generate radial probability density functions, $\mathrm{p}(\mathrm{r})$, and the trajectories are used to determine the particle velocity. The results for the PDFs are shown in Fig. 3 . Particles with $D / d=20$ accumulate, as expected, in a stable annulus at approximately $0.8 \mathrm{R}$ from the centre of the pipe for $R_{b}=350$ - the 'Segré-Silberberg' annulus. For higher Reynolds numbers, particles accumulated preferentially at $t w o$ radial locations: $0.8 \mathrm{R}$ and $0.6 \mathrm{R}$ from the pipe centre. The latter is referred to as the 'inner annulus'. From 300D to 500D downstream, the percentage of particles in this inner annulus decreases, as more and more particle migrate to the equilibrium position. The migration of particles to the equilibrium location slows down with increase in Reynolds number; the lowest $R e_{b}$ case (blue curve) is closest to the equilibrium, while the highest case would require an even longer pipe to fully reach this equilibrium.

Fig, 3 also confirms that the equilibrium position shifts outward with increasing $\operatorname{Re}_{b}$ (Asmolov, 1999). This will be discussed in detail later.
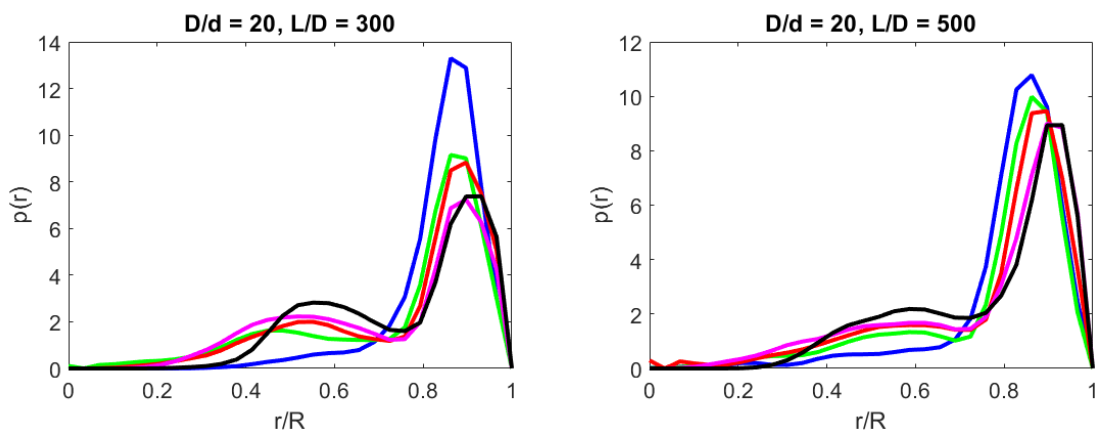

Figure 3: Probability density function $\mathrm{p}(\mathrm{r})$ for particles with $\mathrm{D} / \mathrm{d}=20$. (Left) At 300D from the entrance. (Right) 500D from the entrance. The color represents PDFs for different Reynolds numbers with $R e_{b}=350$ (blue), $R e_{b}=500$ (green), $R e_{b}=800$ (red), $R e_{b}$ $=1000$ (magenta), $R e_{b}=1250$ (black). 

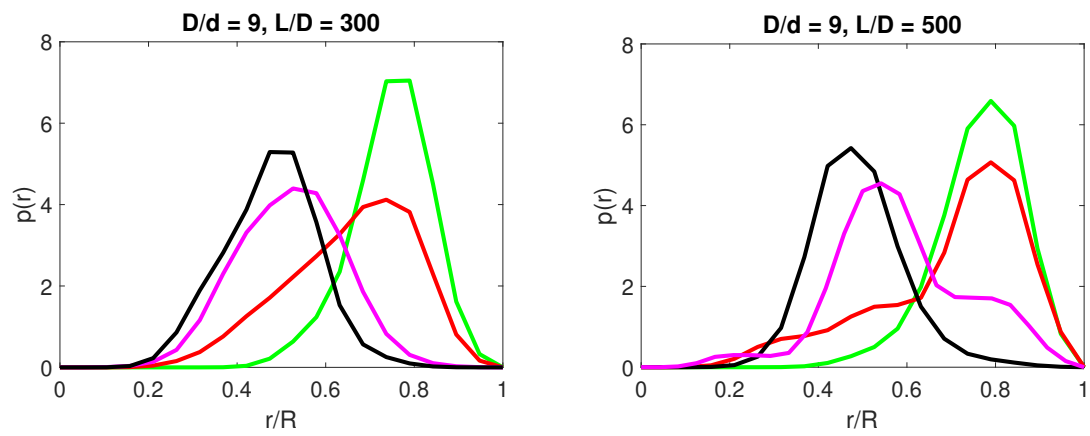

Figure 4: Probability density function $\mathrm{p}(\mathrm{r})$ for particles with $\mathrm{D} / \mathrm{d}=9$. (Left) At 300D from the entrance. (Right) 500D from the entrance. (Color: Fig 3 .

The situation is different for the larger particles $(D / d=6,9)$, where the preferential location appears to depend on the Reynolds number. The particles preferentially appear in the inner annulus at higher Reynolds numbers: at the lowest Reynolds number measured $\left(R e_{b}=350\right)$, they accumulate at $0.8 \mathrm{R}$. For higher Reynolds numbers $\left(\operatorname{Re}_{b}>700\right.$ ), they accumulate at $0.6 \mathrm{R}$ (Fig. 4). The pdfs for particles with D/d $=9$ shows a gradual decrease in the accumulation of particles towards the centre as $R e_{b}$ increases and distributes around one peak. The particles with $\mathrm{D} / \mathrm{d}=6$ show a similar behaviour but accumulate at two preferred locations. The peak positions for different particles at different $R e_{b}$ is shown in Figure 5, along with data from literature.

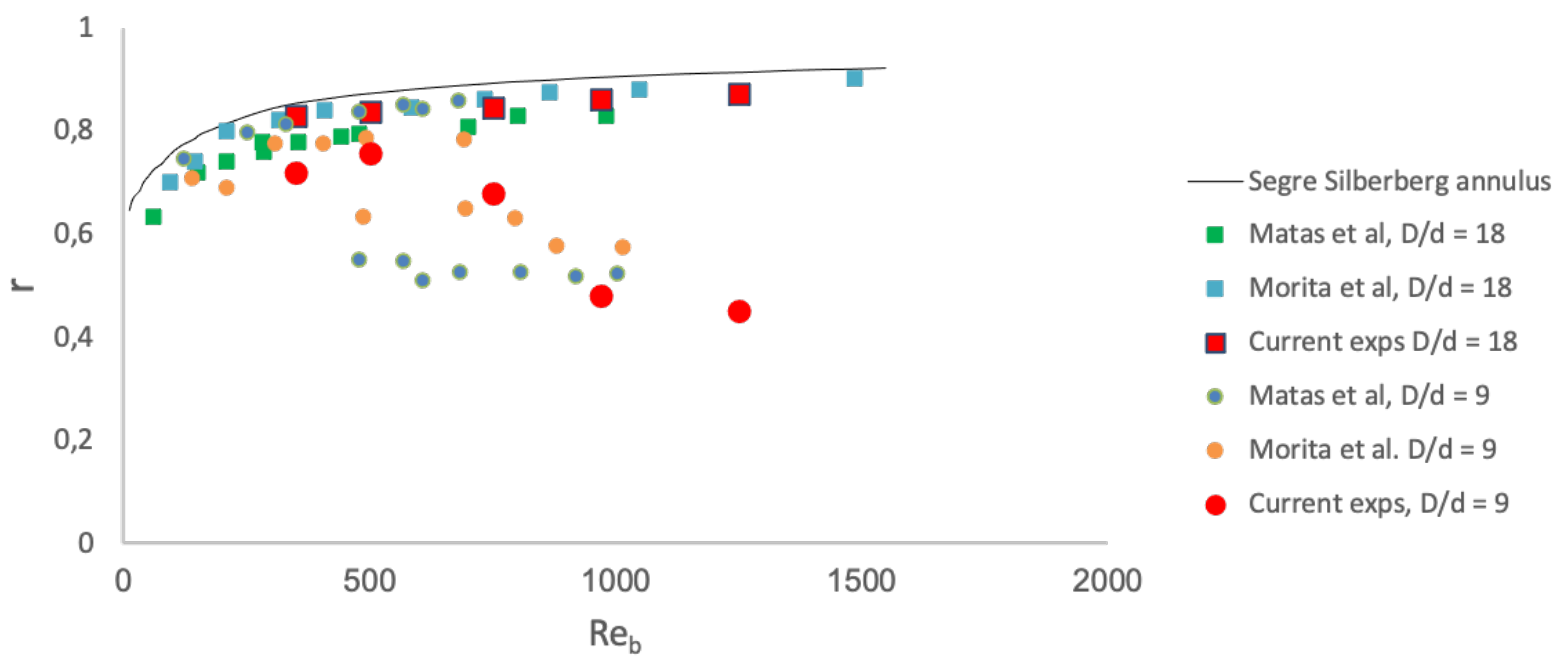

Figure 5: Preferential radial locations of particle accumulation as a function of Reynolds number $\left(\operatorname{Re}_{b}\right)$.

From the reconstructed trajectories, the streamwise velocity of particles match the local fluid velocity with $2 \%$ error margin, rising upto $5 \%$ near wall. Particles at $R e_{b}=350$ are observed to travel mostly parallel to the pipe centreline, with a few particles migrating slowly in the radial direction. At higher Reynolds numbers, particles with $D / d=6$ and 9 are found to be moving in the azimuthal direction, in addition to radial migration. These motions are observed at $0.55-0.85 \mathrm{R}$ from the tube axis, where the azimuthal velocity is higher for particles away from the axis. Statistically, there is no preferred azimuthal direction (clockwise vs. anticlockwise). The azimuthal velocity increases with the Reynolds number and the particle size, with a magnitude less than $5 \%$ of its streamwise velocity $\left(u_{\theta, p} / u_{z, p}<0.05\right)$. At $R e_{b}=1250$, most particles $(85$ $\%$ ) with $\mathrm{D} / \mathrm{d}=9$ are observed to move azimuthally $300 \mathrm{D}$ away from the entrance (Fig. 6). The maximum observed magnitude of the azimuthal velocity of particles is $0.7 \mathrm{~mm} / \mathrm{s}$. At this Reynolds number, particles with $D / d=6$ (Fig 7) have a maximum azimuthal velocity of $3 \mathrm{~mm} / \mathrm{s}$, where they are observed to move over $270^{\circ}$ in $40 \mathrm{D}$ (i.e. throughout the full field of view). Visual inspection confirms that azimuthal motion persists over the entire length of the pipe. This azimuthal (or spiralling) behaviour has been observed before 
(Shao et al. 2008), but little is known about the physical mechanisms behind it.
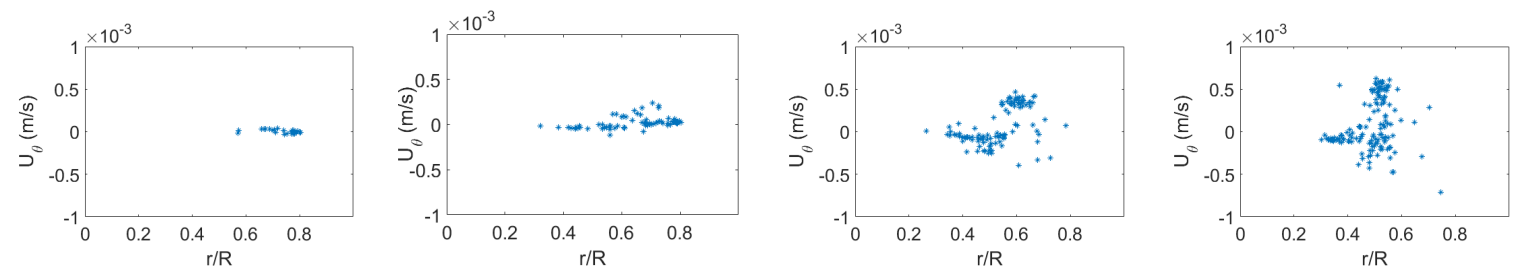

Figure 6: Azimuthal velocity of particles with $\mathrm{D} / \mathrm{d}=9\left(u_{\theta}\right)$ as a function of their radial locations. $R e_{b}=500,750,1000,1250 ; R e_{p}=6.2,8.6$, 12.3, 15.4 (left-right).
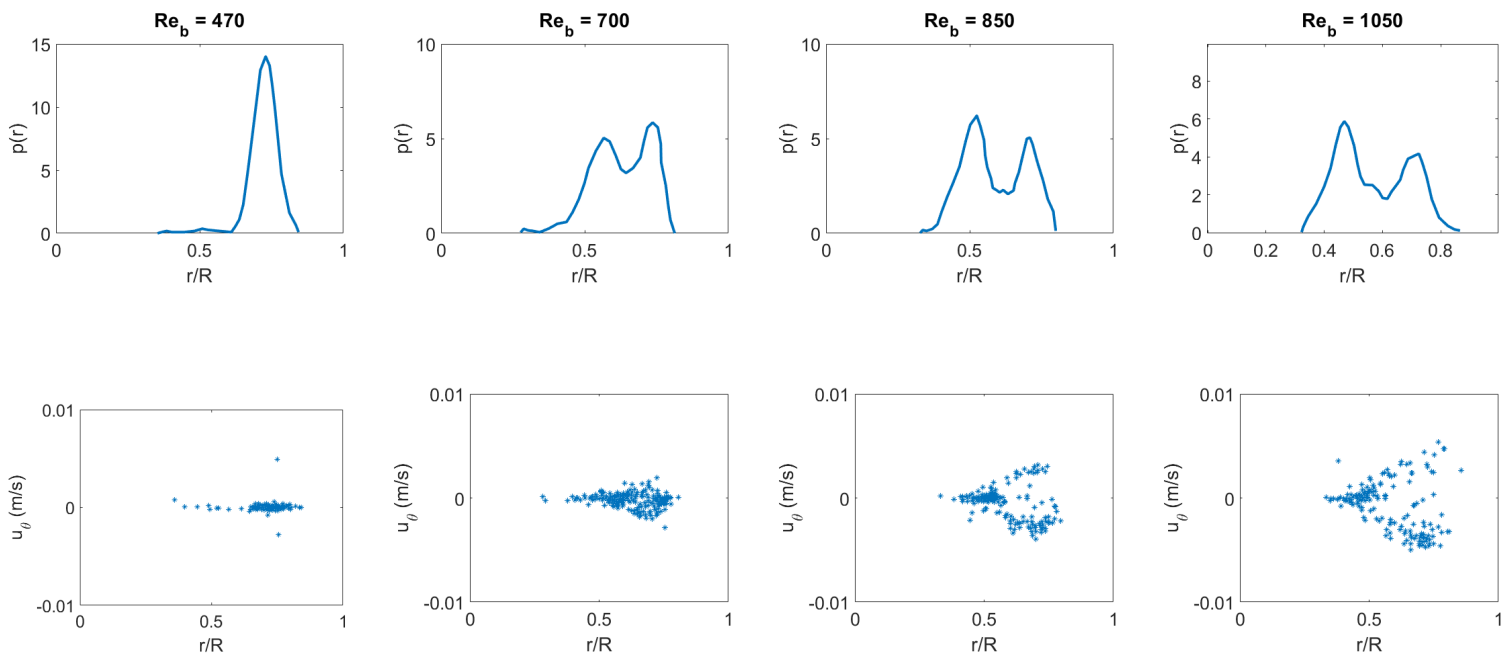

Figure 7: Cross-stream migration observed for particles with $D / d=6$ for different Reynolds numbers (in columns). The top row shows the normalized radial probability density functions, $p(r)$. The bottom row shows the azimuthal velocity $\left(u_{\theta}\right)$ of particles along the radial location of the particles. $R e_{p}=12.5,19.4,23.6$, and 29.1(left to right).

\section{Conclusion and Outlook}

Suspended particles are tracked along several diameters (up to 45D) in three dimensions to study their migration. The reconstruction of 3D trajectories over a narrow and long field of view is demonstrated. The mirror optics contributes to an addition linear mismatch which can be corrected given the angle of view is small $\left(<15^{\circ}\right)$.
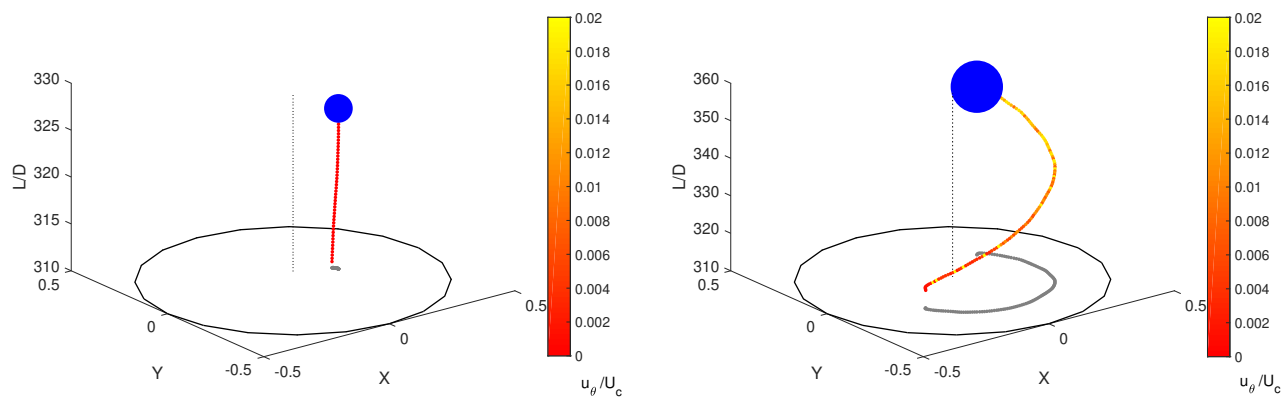

Figure 8: Particle trajectories reconstructed in three-dimensional space at $R e_{b}=600$. The flow direction is from bottom to top. The colormap denotes the azimuthal velocity $\left(u_{\theta}\right)$ of the particle normalized with the centreline velocity $\left(U_{c}\right)$. The circle indicates the flow domain and the gray line represents the projection of tracks in the $(x-y)$ plane. 
The PDFs of radial location constructed are consistent with the literature (Matas et al. (2004); Nakayama et al. (2019); Morita et al. (2017)). The radial displacements are very low, typically under 10 pixels over 20D. For the particles with $D / d=20$, the percentage of particles in the inner annulus decrease downstream. This suggests that the particles would eventually reach the stabe annulus around $0.8 \mathrm{R}$. Large neutrally buoyant particles (D/d $=6$ and 9$)$ migrate radially to a stable annulus as expected (either the inner or outer, depending on the Reynolds number). However, they also move along the azimuthal direction, in contrast to particles of $\mathrm{D} / \mathrm{d}=20$. The azimuthal velocity of the particles increases with the bulk Reynolds number and the particle size. Further, there are also few particles moving inward towards the tube at 300D downstream but the percentage decreases downstream. Such consistent motion of particles show migration patterns that do not agree with theoretical understanding Asmolov (1999). The particle Reynolds number is more than 8 $\left(R e_{p}>8\right)$ for azimuthally moving particles.

Although there is no experimental evidence in the literature, there are two numerical studies (Shao et al., 2008; Yu et al. 2013) that hint at secondary flow patterns. They postulate that the large size of particles leads to azimuthal motion. Note that our results are still not consistent with their work, but the migration patterns at higher $R e_{p}$ does indicate flow reversal around a large particle.

The observation of the azimuthal motion that we report is a result of the fact that we document 3D particles tracks with high fidelity. Previous studies generall relied on planar concentration information (Matas et al. (2004), Morita et al. (2017)).

In ongoing studies, we are investigating the physical background of the peculiar azimuthal motion.

\section{References}

Asmolov ES (1999) The inertial lift on a spherical particle in a plane poiseuille flow at large channel reynolds number. Journal of Fluid Mechanics 381:63-87

Hood K, Lee S, and Roper M (2015) Inertial migration of a rigid sphere in three-dimensional poiseuille flow. Journal of Fluid Mechanics 765:452-479

Matas JP, Morris JF, and Guazzelli É (2004) Inertial migration of rigid spherical particles in poiseuille flow. Journal of Fluid Mechanics 515:171-195

Morita Y, Itano T, and Sugihara-Seki M (2017) Equilibrium radial positions of neutrally buoyant spherical particles over the circular cross-section in poiseuille flow. Journal of Fluid Mechanics 813:750

Nakayama S, Yamashita H, Yabu T, Itano T, and Sugihara-Seki M (2019) Three regimes of inertial focusing for spherical particles suspended in circular tube flows. Journal of Fluid Mechanics 871:952-969

Shao X, Yu Z, and Sun B (2008) Inertial migration of spherical particles in circular poiseuille flow at moderately high reynolds numbers. Physics of Fluids 20:103307

Yu Z, Wu T, Shao X, and Lin J (2013) Numerical studies of the effects of large neutrally buoyant particles on the flow instability and transition to turbulence in pipe flow. Physics of Fluids 25:043305 